\title{
DYNAMICS OF ANTIBIOTIC PRESCRIPTION RATES FOLLOWING PCV IMPLEMENTATION IN CHILDREN <2 YEARS OLD: COMPARISON BETWEEN HIGH AND LOW PRESCRIBING CLINICS
}

Dana Danino ${ }^{1,2}$, Noga Givon-Lavi ${ }^{1,2}$, Shalom Ben-Shimol ${ }^{1,2}$, David Greenberg ${ }^{1,2}$, Ron Dagan $M^{l}$

${ }^{1}$ Faculty of Health Sciences, Ben-Gurion University of the Negev, ${ }^{2}$ The Pediatric Infectious Disease Unit, Soroka University Medical Center, Beer-Sheva, Israel

\section{Introduction}

$$
\text { Results }
$$

Indiscriminate antibiotic use is common among community pediatricians infections (i.e. alveolar pneumonia, otitis media and visits to Emergency Room resulting in chest radiographic examinations) Figure la,b,c. Since respiratory infections are the min reason for antibiotic prescription in young children, we infections (DAPs) will be geations (DAPs) will be greater among high antibiotic-using clinics (HUC) than low antibiotic-using clinics (LUC)

\section{Objectives}

This population-based study was done to:

Determine dynamics of DAPs in children $<2 y$ in relationship to

PCV7/PCV13 implementation

Compare the dynamics between HUC and LUC

Setting

$$
\text { Materials and Methods }
$$

Yearly birth cohort in southern Israel was 14,000 in 2005 and 18,500 in 2016 Compared to Jewish children, Bedouin children live in more crowded conditions, and have higher respiratory morbidity and pneumococcal carriage rates. Before PCV7/PCV13 implementation, antibiotic prescription rates were higher in Bedouin than in Jewish children (Barkai, J Infect Dis, 2004)

$78 \%$ and $86 \%$ of all children $<5$ years of age in Southern Israel district ( in 2005 and 2016, respectively) were insured by Clalit Health Maintenance Organization (Clalit-HMO), where all prescriptions were computerized

PCV7/PCV13 were implemented in July-2009/Nov-2010, respectively, and rapidly reached $\geq 90 \%$ coverage of $\geq 3$ doses

Study design

We enrolled all clinics that: 1) had $\geq 50$ insured children $<5 y$ old and; 2) were active both before and after PCV implementation

Yearly DAP rates were calculated by age ( $<2$ years and $2-4$ years), ethnic groups and antibiotic category (amoxicillin, azithromycin or other antibiotic [cephalosporins, amoxicillin/clavulanate, trimethoprim/sulfamethoxazole, clindamycin, clarithromycin, erythromycin])

Clinics were classified as HUC (above median DAP rates) and LUC (below median DAP rates)

In the pre-PCV years (2005-2009) an average of 9,397 and 7,225 children <2y were insured in HUC and LUC, respectively. The respective figures for children $<5$ y were 12,771 and 32,175

\section{Statistical analysis}

Data were obtained from the computerized HMO databases. Incidences and 95\% CI are presented as the number of prescriptions per 1,000 insured children. A P-value $<0.05$ was considered statistically significant Results

Overall, 965,150 and 486,211 prescriptions were dispensed for children $<5 y$ and $<2$ y, respectively, from July 2005 through June 2016

Amoxicillin was the most frequently prescribed antibiotic (60\%) Figure 2 , followed by azithromycin (12.7\%). These proportions were similar when calculated for HUC vs. LUC, or Bedouin vs. Jewish children

Following PCV7/PCV13 implementation, DAP rate dynamics had a pattern with similarities to that observed for alveolar pneumonia, otitis media and chest $X$ rays Figure 1

PCV implementation resulted in an overall reduction of 1795.1 DAPs per 1000 children during their first 5 years of life (95\% CI 1726.0 to 1864.1 ) and 1402.2 per 1000 children during their 2 years of life (95\% CI 1338.0 to 1465.4) The greatest reduction in DAP rates was observed in children $<2 \mathrm{y}$, thus we decided to focus on this age group Figure $1 d$
Overall mean ( \pm SD) DAP incidence (per 1000 child-years) during pre-PCV implementation was $3567.8 \pm 139.4$ and $1667.8 \pm 42.5$, in the HUC and LUC, respectively $(\mathrm{P}<0.001)$ Figure $3 a$

Before PCV implementation, no significant trend in overall DAP rates was observed; however, comparing 2014-16 to 2005-09, overall DAP rates declined significantly in the HUC ( $33 \%, \mathrm{P}<0.001)$, but not in LUC, Figure 3 It is remarkable that the higher the initial DAPs rate was, the steeper was the decline - thus achieving by 2015-16 a partial closing of the gap between Jewish clinics and Bedouin clinics and between HUC and LUC Figure $3 b$,

The dynamics of amoxicillin DAP rates paralleled those of the overall antibiotics Figure $3 d$

Azithromycin was not reduced after PCV7/PCV13 implementation in HUC, and a significant increase was found in LUC Figure $3 e$

For other antibiotics DAP rates decreased in both HUC and LUC, but the decline was already significant before PCV implementation

Figure 1: Rate Dynamics of Common Respiratory-related Morbidity in Children in Southern Israel, 2005 through 2016
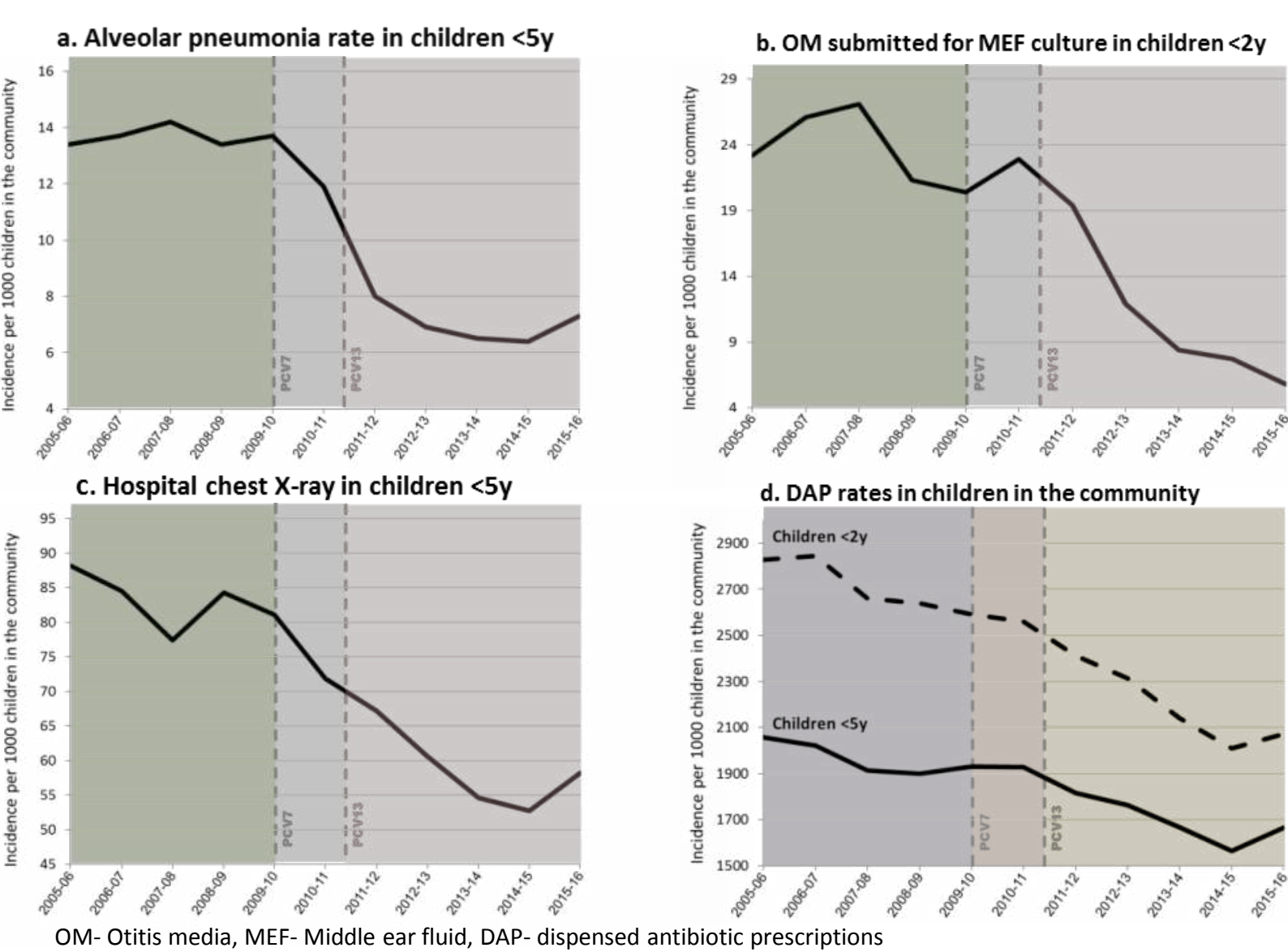

Figure 2: Proportions of dispensed antibiotic prescriptions by antibiotic category, before PCV implementation, July 2005 - June 2009
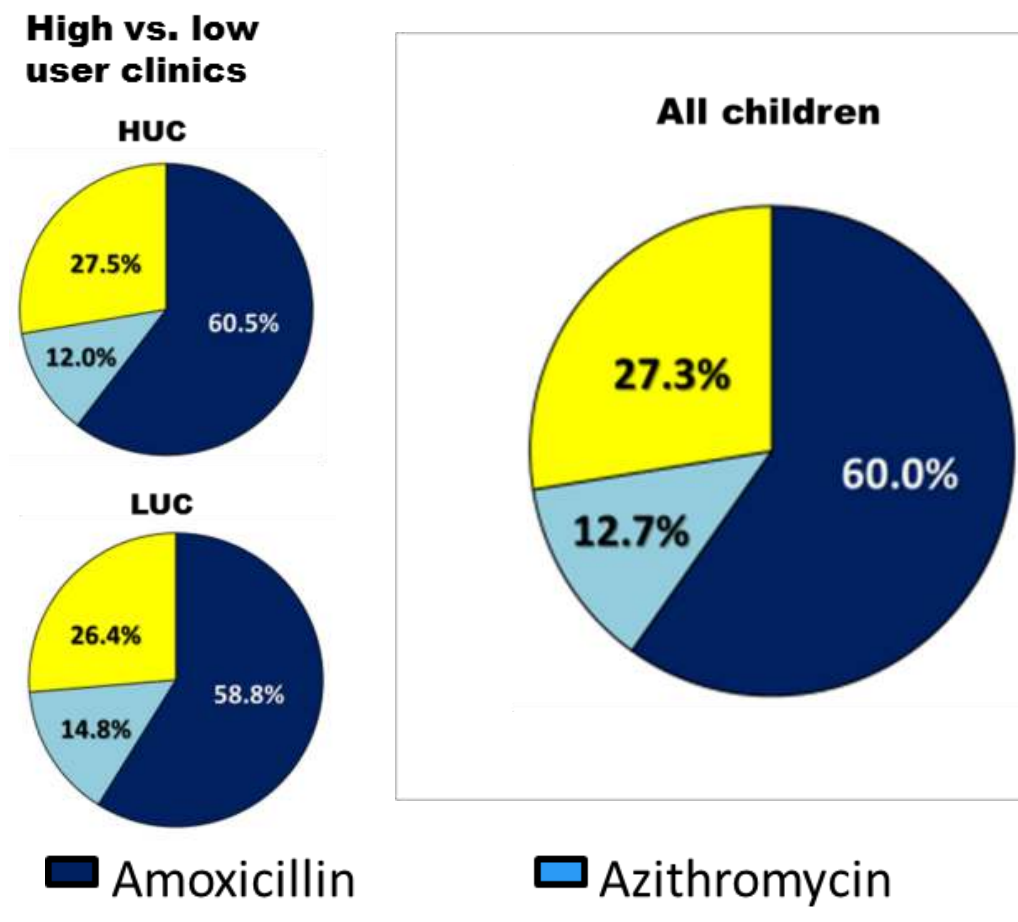

Bedouin vs.
Jewish childre
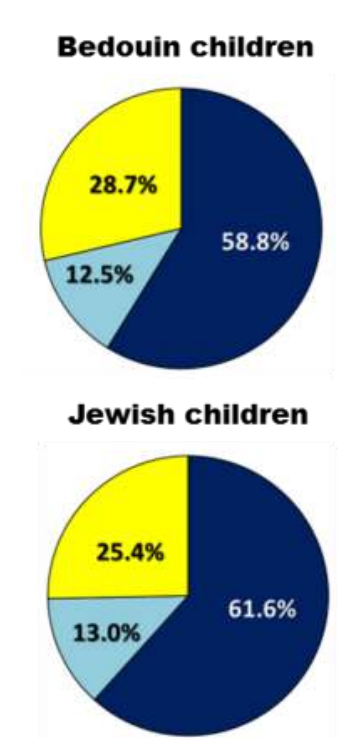

口Azithromycin Results

Figure 3: Antibiotic prescription rates in children $<2 \mathrm{yr}$, in all, high and low antibiotic prescribing clinics, July 2005 through June 2016
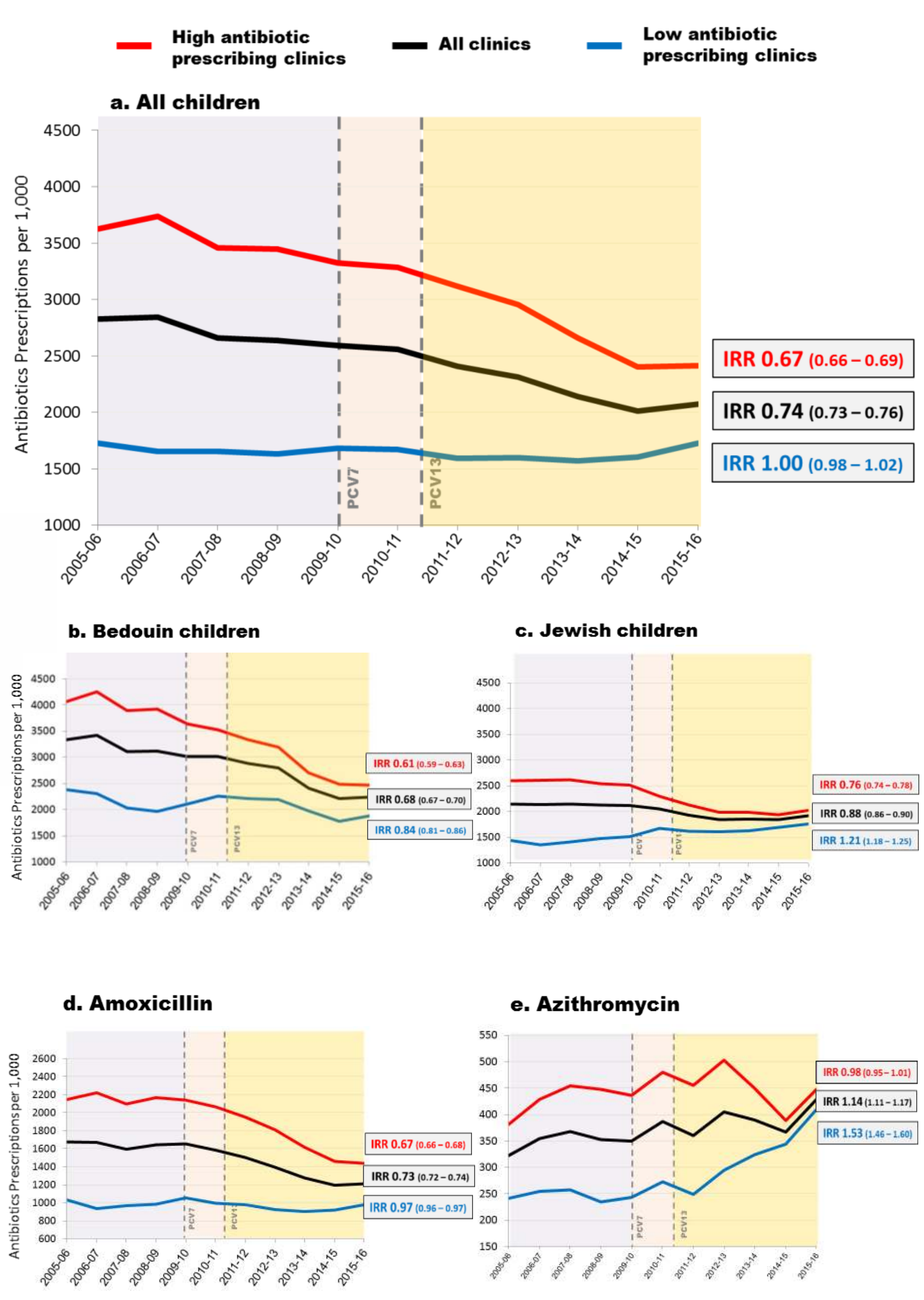

Incidence Rate Ratio (IRRs) $\pm 95 \%$ CI (July 2014-June 2016 vs. July 2005-June 2009)

Conclusions

A significant reduction in DAP rates was observed in children in southern Israel, in association with PCV7/PCV13 implementation

PCV7/PCV13 implementation was associated with a significant decline in HUC DAP rates but not LUC DAP rates, resulting in partial closing of the DAP rates gap between HUC and LUC

Similar trends were found in both distinct ethnic groups (Jewish and Bedouin children) despite significantly higher DAP rates in Bedouin children than Jewish children in the pre-PCV era 